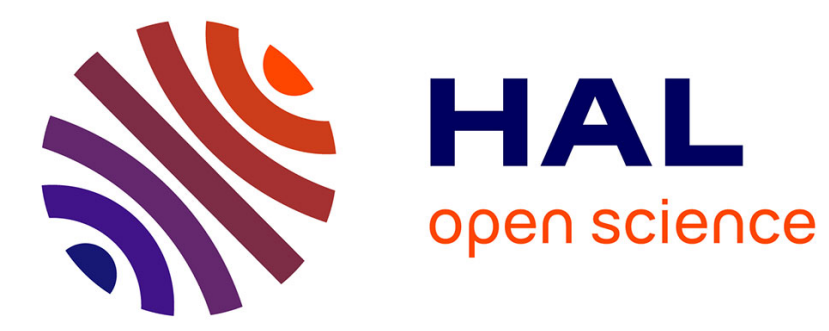

\title{
p-Laplacian regularization of signals on directed graphs
}

Zeina Abu Aisheh, Sébastien Bougleux, Olivier Lézoray

\section{To cite this version:}

Zeina Abu Aisheh, Sébastien Bougleux, Olivier Lézoray. p-Laplacian regularization of signals on directed graphs. International Symposium on Visual Computing, Nov 2018, Las Vegas, United States. pp.650-661. hal-01923318

\section{HAL Id: hal-01923318 \\ https://hal.science/hal-01923318}

Submitted on 15 Nov 2018

HAL is a multi-disciplinary open access archive for the deposit and dissemination of scientific research documents, whether they are published or not. The documents may come from teaching and research institutions in France or abroad, or from public or private research centers.
L'archive ouverte pluridisciplinaire HAL, est destinée au dépôt et à la diffusion de documents scientifiques de niveau recherche, publiés ou non, émanant des établissements d'enseignement et de recherche français ou étrangers, des laboratoires publics ou privés. 


\title{
$p$-Laplacian regularization of signals on directed graphs $^{\star}$
}

\author{
Zeina Abu Aisheh, Sébastien Bougleux, and Olivier Lézoray \\ Normandie Univ, UNICAEN, ENSICAEN, CNRS, GREYC, 14000 Caen, France \\ \{zeina.abu-aisheh, sebastien.bougleux, olivier.lezoray\}@unicaen.fr
}

\begin{abstract}
The graph Laplacian plays an important role in describing the structure of a graph signal from weights that measure the similarity between the vertices of the graph. In the literature, three definitions of the graph Laplacian have been considered for undirected graphs: the combinatorial, the normalized and the random-walk Laplacians. Moreover, a nonlinear extension of the Laplacian, called the $p$-Laplacian, has also been put forward for undirected graphs. In this paper, we propose several formulations for $p$-Laplacians on directed graphs directly inspired from the Laplacians on undirected graphs. Then, we consider the problem of $p$-Laplacian regularization of signals on directed graphs. Finally, we provide experimental results to illustrate the effect of the proposed $p$-laplacians on different types of graph signals.
\end{abstract}

Keywords: Directed graphs $\cdot p$-Laplacian · Graph signal · Regularization.

\section{Introduction}

With the development of new sensors, signals can now be generated from many different sources providing images, meshes, social or biological networks, to quote a few. These signals often have a structure much more irregular than a classical Euclidean grid but they can be represented by graphs [9]. When data vectors are associated with graph vertices, a so-called graph signal is obtained. The new research field of signal processing on graphs aims at extending the classical discrete signal processing tools to signals living on an underlying irregular graph (see [14] for a review) and one of its key ingredients is the graph Laplacian. Indeed, the graph Laplacian plays an important role in describing the structure of a graph signal from weights that measure the similarity between the vertices of the graph [3]. Several definitions of the graph Laplacian have been considered so far for undirected graphs [10]: the combinatorial Laplacian, the normalized Laplacian and the random-walk Laplacian. These Laplacians have become increasingly popular in graph signal processing $[13,8,16]$ and machine learning

\footnotetext{
* This work received funding from the Agence Nationale de la Recherche (ANR-14CE27-0001 GRAPHSIP), and from the European Union FEDER/FSE 2014/2020 (GRAPHSIP project).
} 
$[11,10]$. Some extensions of the Laplacian have been also proposed for directed graphs $[4,1,7]$. A nonlinear extension of the Laplacian also exists that is called the $p$-Laplacian. This latter operator has been considered for undirected graphs and enables to recover analogues (when $p=2$ ) of the combinatorial Laplacian $[2,6]$ and of the normalized Laplacian [18]. Unfortunately there exists actually no formulation of the $p$-Laplacian for the general case of directed graphs and we propose in this paper three different formulations for the latter (i.e., combinatorial, normalized, and random-walk, similarly to the Laplacian on undirected graphs). Then, we consider the problem of $p$-Laplacian regularization of signals on directed graphs that minimizes a loss function plus a regularization term. To the best of our knowledge this is the first time that the use of the $p$-Laplacian on directed graphs is considered for graph signal regularization. Finally we show some results for the filtering of different graph signals.

\section{Notations}

We introduce in this section the notations that will be used in the paper. A graph represents a set of elements and a set of pairwise relationships between those elements [9]. The elements are called vertices and the relationships are called edges. Formally, a graph $\mathcal{G}[5]$ is defined by the sets $\mathcal{G}=(\mathcal{V}, \mathcal{E})$ in which $\mathcal{E} \subseteq \mathcal{V} \times \mathcal{V}$. We denote the $i$ th vertex as $v_{i} \in \mathcal{V}$. Since each edge is a subset of two vertices, we write $e_{i j}=\left\{v_{i}, v_{j}\right\}$. A graph is called directed when each edge $e_{i j}$ contains an ordering of the vertices. A directed edge from $v_{j}$ to $v_{i}$ will be denoted $v_{j} \rightarrow v_{i}$. The edges of a graph can be weighted with a function denoted by $w: \mathcal{E} \rightarrow \mathbb{R}^{+}$. The adjacency matrix representation of directed graph is a $|\mathcal{V}| \times|\mathcal{V}|$ matrix $\mathbf{W}$ where $\mathbf{W}_{i j}=w\left(v_{i}, v_{j}\right)$ if $v_{i} \rightarrow v_{j} \in \mathcal{E}$ and 0 otherwise. For undirected graphs the matrix $\mathbf{W}$ is symmetric and $\mathbf{W}^{T}=\mathbf{W}$. This will not be the case in this paper since we consider directed graphs and if $v_{i} \rightarrow v_{j} \in \mathcal{E}$ this will not necessarily imply that $v_{j} \rightarrow v_{i} \in \mathcal{E}$ or that $w\left(v_{i}, v_{j}\right)=w\left(v_{j}, v_{i}\right)$. The out-degree of a node $v_{i}, \mathrm{~d}^{+}\left(v_{i}\right)$, is equal to $\mathrm{d}^{+}\left(v_{i}\right)=\sum_{v_{i} \rightarrow v_{j} \in \mathcal{E}} w_{i j}$. The in-degree of a node $v_{i}, \mathrm{~d}^{-}\left(v_{i}\right)$, is equal to $\mathrm{d}^{-}\left(v_{i}\right)=\sum_{v_{j} \rightarrow v_{i} \in \mathcal{E}} w_{j i}$. Note that in an undirected graph, $\mathrm{d}^{+}\left(v_{i}\right)=\mathrm{d}^{-}\left(v_{i}\right), \forall v_{i} \in \mathcal{V}$ and is denoted $\mathrm{d}\left(v_{i}\right)$. The out-degree matrix $\mathbf{D}_{+}$is a diagonal matrix with $\mathbf{D}_{+i i}=\mathrm{d}^{+}\left(v_{i}\right)$, and similarly for $\mathbf{D}_{-}$. When the graph is undirected, one has $\mathbf{D}_{+}=\mathbf{D}_{-}=\mathbf{D}$. For undirected graphs, several Laplacian formulation exist [10]. The combinatorial Laplacian is the matrix $\mathbf{L}=\mathbf{D}-\mathbf{W}$. The normalized Laplacian is $\tilde{\mathbf{L}}=\mathbf{D}^{-1 / 2} \mathbf{L} \mathbf{D}^{-1 / 2}=$ $\mathbf{I}-\mathbf{D}^{-1 / 2} \mathbf{W} \mathbf{D}^{-1 / 2}$. The random walk Laplacian is $\mathbf{L}^{r w}=\mathbf{D}^{-1} \mathbf{L}=\mathbf{I}-\mathbf{D}^{-1} \mathbf{W}$. Now we define the space of functions on graphs (i.e., for graph signals). Let $\mathcal{H}(\mathcal{V})$ be the Hilbert space of real-valued functions defined on the vertices of a graph, a graph signal is a function $f: \mathcal{V} \rightarrow \mathbb{R}^{n}$ of $\mathcal{H}(\mathcal{V})$ that maps each vertex to a vector $\mathbf{f}\left(v_{i}\right)$. The space $\mathcal{H}(\mathcal{V})$ is endowed with the usual inner product $\langle f, h\rangle_{\mathcal{H}(\mathcal{V})}=\sum_{v_{i} \in \mathcal{V}} f\left(v_{i}\right) h\left(v_{i}\right)$, where $f, h: \mathcal{V} \rightarrow \mathbb{R}$. Similarly, let $\mathcal{H}(\mathcal{E})$ be the space of real-valued functions defined on the edges of $\mathcal{G}$. It is endowed with the inner product $\langle F, H\rangle_{\mathcal{H}(\varepsilon)}=\sum_{e_{i j} \in \mathcal{E}} F\left(e_{i j}\right) H\left(e_{i j}\right)$, where $F, H: \mathcal{E} \rightarrow \mathbb{R}$ are two functions of $\mathcal{H}(\varepsilon)$. 


\section{$3 \quad p$-Laplacian on directed graphs}

\subsection{Definitions}

To define the $p$-Laplacian we need first to introduce several operators that operate on directed graphs. The formulation of these operators is similar to the one found in [18] but are expressed here on directed graphs. It is important to note that in contrast to undirected graphs, there has been few studies on Laplacian for directed graphs [4] and even less for the $p$-Laplacian [19]. The directed difference operator of a graph signal $f \in \mathcal{H}(\mathcal{V})$, called $d_{w}: \mathcal{H}(\mathcal{V}) \rightarrow \mathcal{H}(\mathcal{E})$, over a directed edge $v_{i} \rightarrow v_{j}$ is denoted by $\left(d_{w} f\right)\left(v_{i}, v_{j}\right)$. We do not explicitly provide now the definition of this difference operator and will show that with different definitions, different $p$-Laplacian on directed graphs can be formulated. The adjoint operator $d_{w}^{*}: \mathcal{H}(\mathcal{E}) \rightarrow \mathcal{H}(\mathcal{V})$, of a function $H \in \mathcal{H}(\mathcal{E})$, can then be expressed at a vertex $v_{i} \in \mathcal{V}$ by using the definition of the inner products since $\left\langle H, d_{w} f\right\rangle_{\mathcal{H}(\mathcal{E})}=\left\langle d_{w}^{*} H, f\right\rangle_{\mathcal{H}(\mathcal{V})}$. The gradient operator of a function $f \in \mathcal{H}(\mathcal{V})$, at vertex $v_{i} \in \mathcal{V}$, is the vector of all the weighted directed differences $\left(d_{w} f\right)\left(v_{i}, v_{j}\right)$, with respect to the set of outgoing edges $v_{i} \rightarrow v_{j} \in \mathcal{E}$ :

$$
\left(\nabla_{\mathbf{w}} \mathbf{f}\right)\left(v_{i}\right)=\left(\left(d_{w} f\right)\left(v_{i}, v_{j}\right)\right)^{T}, \forall\left(v_{i} \rightarrow v_{j}\right) \in \mathcal{E} .
$$

Its $\mathcal{L}_{p}$ norm is defined by

$$
\left\|\left(\nabla_{\mathbf{w}} \mathbf{f}\right)\left(v_{i}\right)\right\|_{p}=\left[\sum_{v_{i} \rightarrow v_{j} \in \mathcal{E}}\left|\left(d_{w} f\right)\left(v_{i}, v_{j}\right)\right|^{p}\right]^{1 / p} .
$$

Then, the $p$-Laplacian $\Delta_{w}^{p} f: \mathcal{H}(\mathcal{V}) \rightarrow \mathcal{H}(\mathcal{V})$ can be formulated as the discrete analogue of the continuous one by $[6]$ :

$$
\Delta_{w}^{p} f\left(v_{i}\right)=\frac{1}{2} d_{w}^{*}\left(\left\|\nabla_{\mathbf{w}} \mathbf{f}\left(v_{i}\right)\right\|_{2}^{p-2}\left(d_{w} f\right)\left(v_{i}, v_{j}\right)\right)=\frac{1}{2} d_{w}^{*}\left(\frac{\left(d_{w} f\right)\left(v_{i}, v_{j}\right)}{\left\|\boldsymbol{\nabla}_{\mathbf{w}} \mathbf{f}\left(v_{i}\right)\right\|_{2}^{2-p}}\right)
$$

where $p \in(0,+\infty)$. If we choose specific formulations for the directed difference operator, we can end-up with new formulations of the $p$-Laplacian on directed graphs, that we propose in the sequel. Details are provided only for the first formulation due to paper length constraints.

\subsection{Combinatorial $p$-Laplacian on directed graphs}

First we consider

$$
\left(d_{w} f\right)\left(v_{i}, v_{j}\right)=w\left(v_{i}, v_{j}\right)\left(f\left(v_{j}\right)-f\left(v_{i}\right)\right)
$$

as a directed difference operator on the edge $v_{i} \rightarrow v_{j}$. This is similar to the one used for the combinatorial Laplacian on undirected graphs. Using the definitions of the inner products in $\mathcal{H}(\mathcal{E})$ and $\mathcal{H}(\mathcal{V})$, we can express the adjoint operator

$$
\left(d_{w}^{*} H\right)\left(v_{i}\right)=\sum_{v_{j} \rightarrow v_{i}} H\left(v_{j}, v_{i}\right) w\left(v_{j}, v_{i}\right)-\sum_{v_{i} \rightarrow v_{j}} H\left(v_{i}, v_{j}\right) w\left(v_{i}, v_{j}\right) .
$$


Proof.

$$
\begin{aligned}
& \left\langle H, d_{w} f\right\rangle_{\mathcal{H}(E)}=\sum_{\left(v_{i}, v_{j}\right) \in E}\left(d_{w} f\right)\left(v_{i}, v_{j}\right) H\left(v_{i}, v_{j}\right)=\sum_{\left(v_{i}, v_{j}\right) \in E} w\left(v_{i}, v_{j}\right)\left(f\left(v_{j}\right)-f\left(v_{i}\right)\right) H\left(v_{i}, v_{j}\right) \\
& =\frac{1}{2} \sum_{v_{i} \in V}\left(\sum_{v_{i} \rightarrow v_{j}} H\left(v_{i}, v_{j}\right) w\left(v_{i}, v_{j}\right)\left(f\left(v_{j}\right)-f\left(v_{i}\right)\right)+\sum_{v_{j} \rightarrow v_{i}} H\left(v_{j}, v_{i}\right) w\left(v_{j}, v_{i}\right)\left(f\left(v_{i}\right)-f\left(v_{j}\right)\right)\right) \\
& =\frac{1}{2} \sum_{v_{i} \in V}\left(2 \sum_{v_{j} \rightarrow v_{i}} H\left(v_{j}, v_{i}\right) w\left(v_{j}, v_{i}\right) f\left(v_{i}\right)-2 \sum_{v_{i} \rightarrow v_{j}} H\left(v_{i}, v_{j}\right) w\left(v_{i}, v_{j}\right) f\left(v_{i}\right)\right) \\
& =\sum_{v_{i} \in V} f\left(v_{i}\right)\left(\sum_{v_{j} \rightarrow v_{i}} H\left(v_{j}, v_{i}\right) w\left(v_{j}, v_{i}\right)-\sum_{v_{i} \rightarrow v_{j}} H\left(v_{i}, v_{j}\right) w\left(v_{i}, v_{j}\right)\right)=\left\langle d_{w}^{*} H, f\right\rangle_{\mathcal{H}(V)}
\end{aligned}
$$

The adjoint operator measures the difference between the in- and outgoing flows at a vertex and can be associated to the divergence operator denoted by $-d_{w}^{*}$. Then, using Equation (3) and the definitions of $d_{w}, d_{w}^{*}$ and $\left\|\nabla_{\mathbf{w}}\right\|$, a combinatorial $p$-Laplacian formulation on directed graphs can be expressed by:

$$
\begin{array}{r}
\Delta_{w}^{p} f\left(v_{i}\right)=\frac{1}{2}\left(f\left(v_{i}\right)\left(\sum_{v_{j} \rightarrow v_{i}} \frac{w\left(v_{j}, v_{i}\right)^{2}}{\left\|\boldsymbol{\nabla}_{\mathbf{w}} \mathbf{f}\left(v_{j}\right)\right\|_{2}^{2-p}}+\sum_{v_{i} \rightarrow v_{j}} \frac{w\left(v_{i}, v_{j}\right)^{2}}{\left\|\boldsymbol{\nabla}_{\mathbf{w}} \mathbf{f}\left(v_{i}\right)\right\|_{2}^{2-p}}\right)\right. \\
-\left(\sum_{v_{j} \rightarrow v_{i}} \frac{w\left(v_{j}, v_{i}\right)^{2}}{\left\|\boldsymbol{\nabla}_{\mathbf{w}} \mathbf{f}\left(v_{j}\right)\right\|_{2}^{2-p}} f\left(v_{j}\right)+\sum_{v_{i} \rightarrow v_{j}} \frac{w\left(v_{i}, v_{j}\right)^{2}}{\left.\left.\left\|\boldsymbol{\nabla}_{\mathbf{w}} \mathbf{f}\left(v_{i}\right)\right\|_{2}^{2-p} f\left(v_{j}\right)\right)\right)}\right.
\end{array}
$$

On the opposite to the classical formulation on undirected graphs $[2,6]$, the latter takes into account both ingoing and outgoing edges from vertices. However, with a specific (directed or undirected) graph and a given value of $p$, we can recover several state-of-the-art Laplacian formulations (up to a power of 2 on the weights). With undirected graphs, the formulation of [2] is recovered, with symmetric directed graphs the formulation of [7] is recovered. With $p=2$, other formulations have been proposed on directed graphs but they only use in-degrees as $\Delta_{w}^{2} f=\left(\mathbf{D}_{-}-\mathbf{W}\right) f[15]$ or out-degrees as $\Delta_{w}^{2} f=\left(\mathbf{D}_{+}-\mathbf{W}\right) f$ [12]. Our proposal encompasses these. In particular, for $p=2$, Equation (6) can be expressed as $\Delta_{w}^{2} f=\frac{1}{2}\left(\mathbf{D}_{-}+\mathbf{D}_{+}-\mathbf{W}-\mathbf{W}^{T}\right) f$ in matrix expression. With undirected graphs and the classical combinatorial Laplacian $\Delta_{w}^{2} f=\mathbf{L} f$ is recovered [3].

\subsection{Normalized $p$-Laplacian on directed graphs}

Second we consider

$$
\left(d_{w} f\right)\left(v_{i}, v_{j}\right)=w\left(v_{i}, v_{j}\right)\left(\frac{f\left(v_{j}\right)}{\sqrt{\mathrm{d}^{-}\left(v_{j}\right)}}-\frac{f\left(v_{i}\right)}{\sqrt{\mathrm{d}^{+}\left(v_{i}\right)}}\right)
$$

as a directed difference operator on the edge $v_{i} \rightarrow v_{j}$. This is close to the one used for the normalized Laplacian on undirected graphs, except that we normalize 
with both in- and out-going degrees since we consider directed graphs. Using the definitions of the inner products in $\mathcal{H}(\mathcal{E})$ and $\mathcal{H}(\mathcal{V})$, we can express the adjoint operator

$$
\left(d_{w}^{*} H\right)\left(v_{i}\right)=\sum_{v_{j} \rightarrow v_{i}} \frac{H\left(v_{j}, v_{i}\right) w\left(v_{j}, v_{i}\right)}{\sqrt{\mathrm{d}^{-}\left(v_{i}\right)}}-\sum_{v_{i} \rightarrow v_{j}} \frac{H\left(v_{i}, v_{j}\right) w\left(v_{i}, v_{j}\right)}{\sqrt{\mathrm{d}^{+}\left(v_{i}\right)}} .
$$

Then, using Equation (3) and the definitions of $d_{w}, d_{w}^{*}$ and $\left\|\boldsymbol{\nabla}_{\mathbf{w}}\right\|$, a normalized $p$-Laplacian formulation on directed graphs can be expressed by:

$$
\begin{array}{r}
\tilde{\Delta}_{w}^{p} f\left(v_{i}\right)=\frac{1}{2}\left(f\left(v_{i}\right)\left(\sum_{v_{j} \rightarrow v_{i}} \frac{w\left(v_{j}, v_{i}\right)^{2}}{\mathrm{~d}^{-}\left(v_{i}\right)\left\|\boldsymbol{\nabla}_{\mathbf{w}} \mathbf{f}\left(v_{j}\right)\right\|_{2}^{2-p}}+\sum_{v_{i} \rightarrow v_{j}} \frac{w\left(v_{i}, v_{j}\right)^{2}}{\mathrm{~d}^{+}\left(v_{i}\right)\left\|\boldsymbol{\nabla}_{\mathbf{w}} \mathbf{f}\left(v_{i}\right)\right\|_{2}^{2-p}}\right)\right. \\
-\left(\sum_{v_{j} \rightarrow v_{i}} \frac{w\left(v_{j}, v_{i}\right)^{2}\left\|\boldsymbol{\nabla}_{\mathbf{w}} \mathbf{f}\left(v_{j}\right)\right\|_{2}^{p-2}}{\sqrt{\mathrm{d}^{-}\left(v_{i}\right) \mathrm{d}^{+}\left(v_{j}\right)}} f\left(v_{j}\right)+\sum_{v_{i} \rightarrow v_{j}} \frac{w\left(v_{i}, v_{j}\right)^{2}\left\|\boldsymbol{\nabla}_{\mathbf{w}} \mathbf{f}\left(v_{i}\right)\right\|_{2}^{p-2}}{\sqrt{\mathrm{d}^{+}\left(v_{i}\right) \mathrm{d}^{-}\left(v_{j}\right)}} f\left(v_{j}\right)\right)
\end{array}
$$

As previously we can recover several state-of-the-art formulations. With directed graphs, $p=2$ and weights replaced by their square root, the formulation can be reduced to

$$
\tilde{\Delta}_{w}^{2} f\left(v_{i}\right)=f\left(v_{i}\right)-\frac{1}{2}\left(\sum_{v_{j} \rightarrow v_{i}} \frac{w\left(v_{j}, v_{i}\right) f\left(v_{j}\right)}{\sqrt{\mathrm{d}^{-}\left(v_{i}\right) \mathrm{d}^{+}\left(v_{j}\right)}}+\sum_{v_{i} \rightarrow v_{j}} \frac{w\left(v_{i}, v_{j}\right) f\left(v_{j}\right)}{\sqrt{\mathrm{d}^{+}\left(v_{i}\right) \mathrm{d}^{-}\left(v_{j}\right)}}\right)
$$

or as $\left(\mathbf{I}-\frac{1}{2}\left(\mathbf{D}_{-}^{-1 / 2} \mathbf{W} \mathbf{D}_{+}^{-1 / 2}+\mathbf{D}_{+}^{-1 / 2} \mathbf{W}^{T} \mathbf{D}_{-}^{-1 / 2}\right)\right) f$ in matrix form. This formulation is closely related to the normalized Laplacian for symmetric directed graphs proposed in $[7,17]$ and expressed as $\left(\mathbf{I}-\frac{1}{2}\left(\mathbf{D}^{-1 / 2} \mathbf{W} \mathbf{D}^{-1 / 2}+\mathbf{D}^{-1 / 2} \mathbf{W}^{T} \mathbf{D}^{-1 / 2}\right)\right) f$.

As it can be seen they normalize only by $\mathbf{D}$ since the graph is symmetric, in contrast to our approach. A similar remark can be made for the formation of [4] for symmetric directed graphs that are strongly connected. With undirected graphs, our formulation reduces to $\tilde{\Delta}_{w}^{2} f\left(v_{i}\right)=f\left(v_{i}\right)-\sum_{v_{j} \in V} \frac{w\left(v_{j}, v_{i}\right)}{\sqrt{d\left(v_{i}\right) d\left(v_{j}\right)}} f\left(v_{j}\right)$ which is exactly the normalized Laplacian $\left(\mathbf{I}-\mathbf{D}^{-1 / 2} \mathbf{W} \mathbf{D}^{-1 / 2}\right) f$ for undirected graphs [3].

\subsection{Random-walk $p$-Laplacian on directed graphs}

Third we consider

$$
\left(d_{w} f\right)\left(v_{i}, v_{j}\right)=\frac{w\left(v_{i}, v_{j}\right)}{\sqrt{\mathrm{d}^{+}\left(v_{i}\right)}}\left(f\left(v_{j}\right)-f\left(v_{i}\right)\right)
$$

as a directed difference operator on the edge $v_{i} \rightarrow v_{j}$. This is similar to the one used for the random-walk Laplacian on undirected graphs. Using the definitions 
of the inner products in $\mathcal{H}(\mathcal{E})$ and $\mathcal{H}(\mathcal{V})$, we can express the adjoint operator

$$
\left(d_{w}^{*} H\right)\left(v_{i}\right)=\sum_{v_{j} \rightarrow v_{i}} \frac{H\left(v_{j}, v_{i}\right) w\left(v_{j}, v_{i}\right)}{\sqrt{\mathrm{d}^{+}\left(v_{i}\right)}}-\sum_{v_{i} \rightarrow v_{j}} \frac{H\left(v_{i}, v_{j}\right) w\left(v_{i}, v_{j}\right)}{\sqrt{\mathrm{d}^{+}\left(v_{i}\right)}} .
$$

Then, using Equation (3) and the definitions of $d_{w}, d_{w}^{*}$ and $\left\|\nabla_{\mathbf{w}}\right\|$, a random-walk $p$-Laplacian formulation on directed graphs can be expressed by:

$$
\begin{aligned}
\Delta_{w}^{p, r w} f\left(v_{i}\right) & =\frac{1}{2}\left(f\left(v_{i}\right)\left(\sum_{v_{j} \rightarrow v_{i}} \frac{w\left(v_{j}, v_{i}\right)^{2}}{\mathrm{~d}^{+}\left(v_{j}\right)\left\|\boldsymbol{\nabla}_{\mathbf{w}} \mathbf{f}\left(v_{j}\right)\right\|_{2}^{2-p}}+\sum_{v_{i} \rightarrow v_{j}} \frac{w\left(v_{i}, v_{j}\right)^{2}}{\mathrm{~d}^{+}\left(v_{i}\right)\left\|\boldsymbol{\nabla}_{\mathbf{w}} \mathbf{f}\left(v_{i}\right)\right\|_{2}^{2-p}}\right)\right. \\
- & \left.\left(\sum_{v_{j} \rightarrow v_{i}} \frac{w\left(v_{j}, v_{i}\right)^{2}}{\mathrm{~d}^{+}\left(v_{j}\right)\left\|\boldsymbol{\nabla}_{\mathbf{w}} \mathbf{f}\left(v_{j}\right)\right\|_{2}^{2-p}} f\left(v_{j}\right)+\sum_{v_{i} \rightarrow v_{j}} \frac{w\left(v_{i}, v_{j}\right)^{2}}{\mathrm{~d}^{+}\left(v_{i}\right)\left\|\boldsymbol{\nabla}_{\mathbf{w}} \mathbf{f}\left(v_{i}\right)\right\|_{2}^{2-p}} f\left(v_{j}\right)\right)\right)
\end{aligned}
$$

Again, with a specific (directed or undirected) graph and a given value of $p$, we can recover several state-of-the-art Laplacian formulations (up to a power of 2 on the weights). In particular, for $p=2$, Equation (13) can be expressed as $\Delta_{w}^{2, r w} f=\left(\mathbf{I}-\frac{1}{2} \mathbf{D}_{+}^{-1}\left(\mathbf{W}+\mathbf{W}^{T}\right)\right) f$ in matrix expression. With directed graphs and $p=2$ the formulation in matrix expression of [7] is recovered, and with undirected graphs and $p=2$ the classical random-walk Laplacian $\Delta_{w}^{2, r w} f=\mathbf{L}^{r w} f$ is recovered [10]. With $p=2$, a similar formulation for directed graphs has been expressed as $\left(\mathbf{I}-\mathbf{D}_{+}^{-1} \mathbf{W}\right) f$ in $[1]$.

\section{$4 \quad p$-Laplacian Regularization on Directed Graphs}

In the previous section, we have proposed general formulations for combinatorial, normalized and random-walk $p$-Laplacians on directed graphs. From these, we consider the problem of $p$-Laplacian regularization of signals on directed graphs that minimizes a loss function plus a regularization term. Let $f^{0}: \mathcal{V} \rightarrow \mathbb{R}$ be a noisy graph signal of a clean graph signal $g: \mathcal{V} \rightarrow \mathbb{R}$ corrupted by a given noise $n$ such that $f^{0}=g+n$. To recover the uncorrupted function $g$, a commonly used method is to seek for a function $f: \mathcal{V} \rightarrow \mathbb{R}$ which is regular enough on $\mathcal{G}$, and also close enough to $f^{0}$. This inverse problem can be formalized by the minimization of an energy functional, that typically involves a regularization term plus an approximation term (also called loss). The proposed graph $p$-Laplacians can be used to define a regularization functional $R_{w}^{p}: \mathcal{H}(\mathcal{V}) \rightarrow \mathbb{R}^{+}$on directed graphs by

$$
R_{w}^{p, *}(f)=\left\langle\Delta_{w}^{p, *} f, f\right\rangle_{\mathcal{H}(\mathcal{V})}=\left\langle d_{w} f, d_{w} f\right\rangle_{\mathcal{H}(\varepsilon)}=\sum_{v_{i} \in \mathcal{V}}\left\|\left(\nabla_{\mathbf{w}} \mathbf{f}\right)\left(v_{i}\right)\right\|_{2}^{p}
$$

where $\Delta_{w}^{p, *}$ is among equations $(6),(9)$ or (13). Since $R_{w}^{p, *} \geq 0, \Delta_{w}^{p, *}$ is positive semi-definite. From this we consider the following variational problem of $p$-Laplacian regularization on directed graphs

$$
g \approx \min _{f: \mathcal{V} \rightarrow \mathbb{R}}\left\{E_{w}^{p, *}\left(f, f^{0}, \lambda\right)=\frac{1}{p} R_{w}^{p, *}(f)+\frac{\lambda}{2}\left\|f-f^{0}\right\|_{2}^{2}\right\},
$$


where the regularization functional $R_{w}^{p, *}$ can be induced from one of the proposed $p$-Laplacians on directed graphs. When $p \geq 1$, the energy $E_{w}^{p, *}$ is a convex functional of functions of $\mathcal{H}(\mathcal{V})$. To get the solution of the minimizer (15), we consider the following system of equations

$$
\frac{\partial E_{w}^{p, *}\left(f, f^{0}, \lambda\right)}{\partial f\left(v_{i}\right)}=0, \forall v_{i} \in \mathcal{V}
$$

For all the $p$-Laplacians we have proposed, it can be proved that $\frac{1}{p} \frac{\partial R_{w}^{p, *}}{\partial f\left(v_{i}\right)}=$ $2 \Delta_{w}^{p} f\left(v_{i}\right)$ (this is a direct consequence of Equation (14)) and the system of equations is then re-written as follows:

$$
2 \Delta_{w}^{p, *} f\left(v_{i}\right)+\lambda\left(f\left(v_{i}\right)-f^{0}\left(v_{i}\right)\right)=0
$$

By substituting the expression of $\Delta_{w}^{p, *} f\left(v_{i}\right)$ by one of the proposed $p$-Laplacians $\left(\Delta_{w}^{p}, \tilde{\Delta}_{w}^{p}, \Delta_{w}^{p, r w}\right)$ into Equation (17), the system of equations can be solved using a linearized Gauss-Jacobi iterative method. Let $t$ be an iteration step, and $f^{(t)}$ be the solution at step $t$, then the following iterative algorithm is obtained for each of the proposed $p$-Laplacian on directed graphs.

$$
f^{t+1}\left(v_{i}\right)=\frac{\lambda f^{0}\left(v_{i}\right)+\left(\sum_{v_{j} \rightarrow v_{i}} \frac{w\left(v_{j}, v_{i}\right)^{2} f^{t}\left(v_{j}\right)}{\phi\left(v_{j}, v_{i}\right)\left\|\nabla_{\mathbf{w}} \mathbf{f}^{\mathbf{t}}\left(v_{j}\right)\right\|_{2}^{2-p}}+\sum_{v_{i} \rightarrow v_{j}} \frac{\left.w\left(v_{i}, v_{j}\right)^{2}\right) f^{t}\left(v_{j}\right)}{\phi\left(v_{i}, v_{j}\right)\left\|\boldsymbol{\nabla}_{\mathbf{w}} \mathbf{f}^{\mathbf{t}}\left(v_{i}\right)\right\|_{2}^{2-p}}\right)}{\lambda+\sum_{v_{j} \rightarrow v_{i}} \frac{w\left(v_{j}, v_{i}\right)^{2}}{\gamma_{1}\left(v_{j}, v_{i}\right)\left\|\boldsymbol{\nabla}_{\mathbf{w}} \mathbf{f}^{\mathbf{t}}\left(v_{j}\right)\right\|_{2}^{2-p}}+\sum_{v_{i} \rightarrow v_{j}} \frac{w\left(v_{i}, v_{j}\right)^{2}}{\gamma_{2}\left(v_{i}, v_{j}\right)\left\|\boldsymbol{\nabla}_{\mathbf{w}} \mathbf{f}^{\mathbf{t}}\left(v_{i}\right)\right\|_{2}^{2-p}}}
$$

where $\phi, \gamma_{1}$ and $\gamma_{2}$ are defined as follows, depending on the chosen directed $p$-Laplacian $\Delta_{w}^{p, *}$ :

- Combinatorial $p$-Laplacian: $\phi\left(v_{j}, v_{i}\right)=\gamma_{1}\left(v_{j}, v_{i}\right)=\gamma_{2}\left(v_{i}, v_{j}\right)=1$,

- Normalized $p$-Laplacian: $\phi\left(v_{j}, v_{i}\right)=\sqrt{\mathrm{d}^{-}\left(v_{i}\right) \mathrm{d}^{+}\left(v_{j}\right)}, \gamma_{1}\left(v_{j}, v_{i}\right)=\mathrm{d}^{-}\left(v_{i}\right)$ and $\gamma_{2}\left(v_{i}, v_{j}\right)=\mathrm{d}^{+}\left(v_{i}\right)$,

- Random-walk $p$-Laplacian: $\phi\left(v_{i}, v_{j}\right)=\mathrm{d}^{+}\left(v_{i}\right)$ and $\gamma_{1}\left(v_{j}, v_{i}\right)=\gamma_{2}\left(v_{i}, v_{j}\right)=$ $\mathrm{d}^{+}\left(v_{i}\right)$.

\section{Experiments and Results}

In this section we provide sample results for the filtering of three different types of graphs signals for different directed graphs topologies and $p$-Laplacians. PSNR values will be used to compare the results. To weight the edges of the graphs, we use a parameterless function $w\left(v_{i}, v_{j}\right)=1-\frac{\left\|\mathbf{F}_{\tau}^{\mathbf{f}^{0}}\left(v_{i}\right)-\mathbf{F}_{\tau}^{\mathbf{f}^{0}}\left(v_{j}\right)\right\|_{2}}{\max _{v_{k} \rightarrow v_{l} \in \mathcal{E}}\left\|\mathbf{f}_{\tau}^{\mathbf{f}^{0}}\left(v_{k}\right)-\mathbf{F}_{\tau}^{0}\left(v_{l}\right)\right\|_{2}}$. The vector $\mathbf{F}_{\boldsymbol{\tau}}^{\mathbf{f}^{0}}\left(v_{i}\right)=\left(f^{0}\left(v_{j}\right): v_{j} \in \mathcal{N}_{\tau}\left(v_{i}\right) \cup\left\{v_{i}\right\}\right)^{T}$ corresponds to the set of values around $v_{i}$ within a $\tau$-hop $\mathcal{N}_{\tau}\left(v_{i}\right)$ (for images this is a patch of size $\left.(2 \tau+1)^{2}\right)$. We will consider two types of directed graphs: 8-adjacency directed grid graph (denoted 
$\mathcal{G}_{0}$, that connects each vertex to its 8 spatially closest nearest neighbors), and $k$-nearest neighbor directed graphs (denoted $\mathcal{G}_{k}^{\alpha, \tau}$, that connects each vertex to its $k$ nearest neighbors in terms of $\mathbf{F}_{\tau}^{\mathbf{f}^{0}} L_{2}$ norm within a $\alpha$-hop). Figure 1 illustrates the influence of the graph construction: nearest neighbors with patchbased distances better capture the image geometry and this can be beneficial for latter processing.

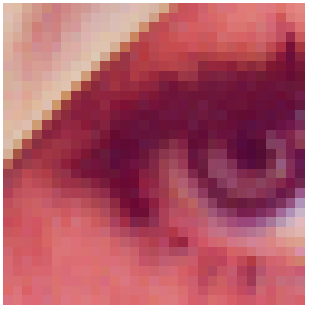

Original image

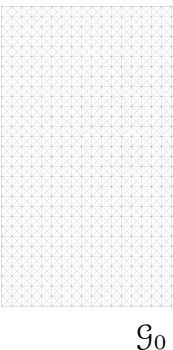

$\mathcal{G}_{0}$

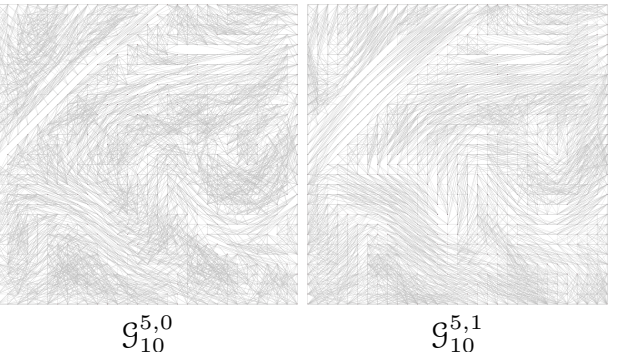

$\mathcal{G}_{10}^{5,0}$
$\mathcal{G}_{10}^{5,1}$

Fig. 1. Examples of directed graphs for an image. From left to right: original image, a symmetric 8-grid graph, 10-nearest neighbor graphs (inside a $11 \times 11$ window with color-based or $3 \times 3$ patch-based distances).

\subsection{Images}

The first type of graph signal we consider is 2D color images (see Figure 2) and $f: \mathcal{V} \rightarrow \mathbb{R}^{3}$. An image has been corrupted by Gaussian noise and we filter the latter with $p$-Laplacian regularization with different configurations: a 8 -adjacency directed grid graph (with $\lambda=0.05, p=1$ ), and a 8-adjacency directed grid graph augmented with a 10-nearest neighbor graph within a 5-hop $(\lambda=0.09$ with patches of size $3 \times 3)$. The nonlinear 1-Laplacian always provides better results than the linear 2-Laplacian. With $\mathcal{G}_{0}$ the graph is directed but symmetric and the filtering reduces to the undirected case [6], whereas with $\mathcal{G}_{0} \cup \mathcal{G}_{10}^{5,1}$ the graph is not symmetric. As it can be seen, adding directed edges to the graph can enhance the results. We have observed that for the directed normalized $p$ Laplacian the results can degrade when low values of the in-degree occur. This is not the case for $\Delta_{w}^{p, r w}$ that always enables to obtain better results whatever the configurations we tested.

\subsection{Database of images}

The second type of graph signal we consider is an image database (see Figure 3) and $f: \mathcal{V} \rightarrow \mathbb{R}^{28 \times 28}$. We selected a subset of 90 images from the MNIST database for digits $0,1,3$ and corrupted the images with Gaussian noise of standard devision $\sigma$. Then a directed 5-nearest neighbor graph is constructed on the whole dataset $(\alpha=\infty)$. This graph is not symmetric. As it can be seen in 

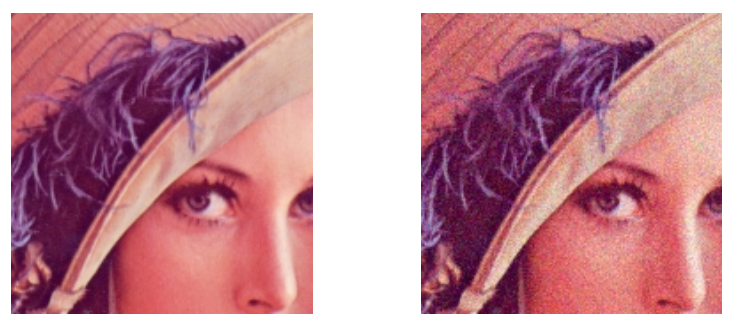

Original Image Corrupted image $(\sigma=15): 24.69 \mathrm{~dB}$
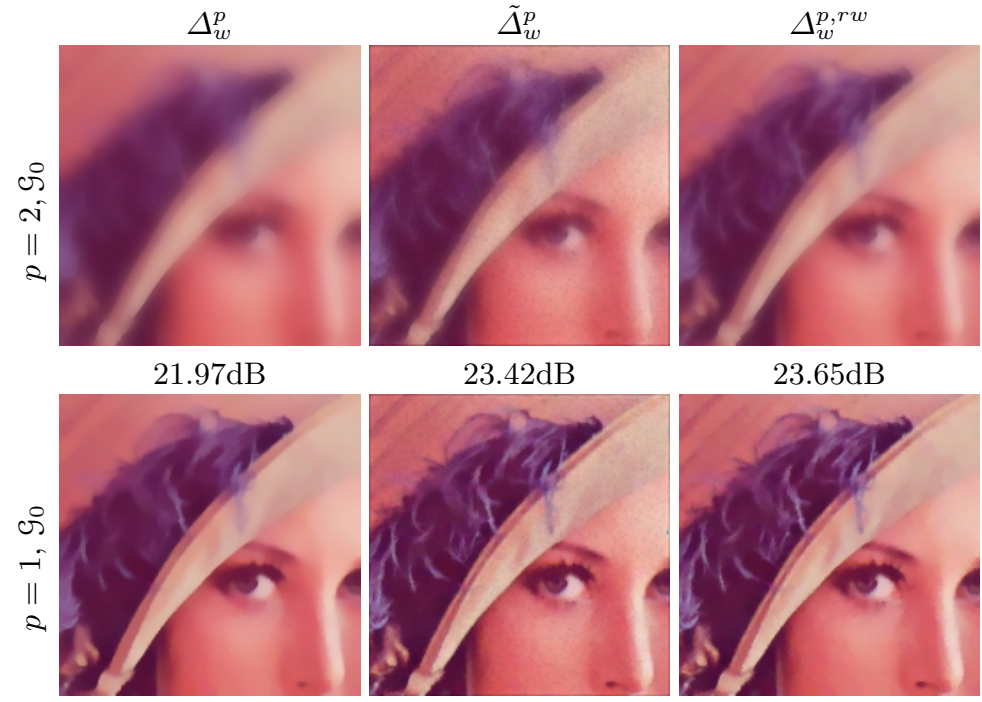

$23.42 \mathrm{~dB}$

$23.65 \mathrm{~dB}$
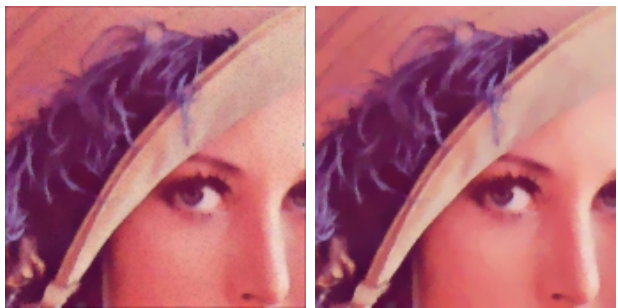

$26.47 \mathrm{~dB}$

$28.79 \mathrm{~dB}$

$29.33 \mathrm{~dB}$

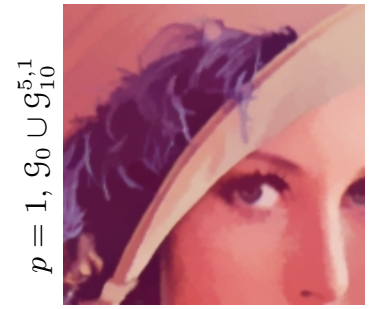

$28.15 \mathrm{~dB}$

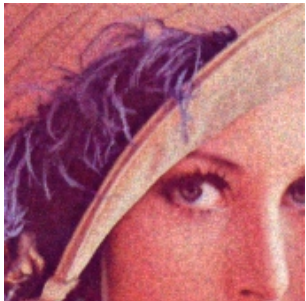

$27.11 \mathrm{~dB}$

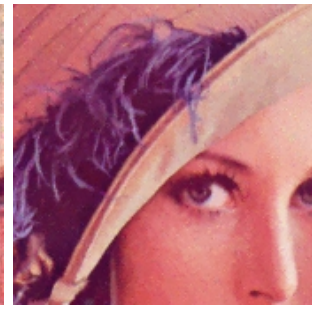

$30.12 \mathrm{~dB}$

Fig. 2. $p$-Laplacian regularization of a corrupted image. See text for details. 
Figure 3, the filtering with the $\tilde{\Delta}_{w}^{1}$ enables to remove the noise while preserving the main structures. Table 1 presents additional results for different amounts of noise. Again, better results are obtained with $p=1$ but the best results are obtained with different $p$-Laplacians, the normalized Laplacian having this time a much better behavior than for images.

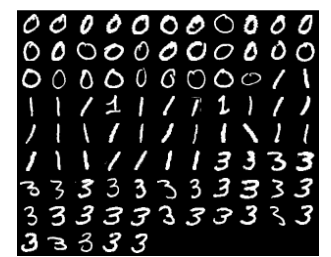

Original DB

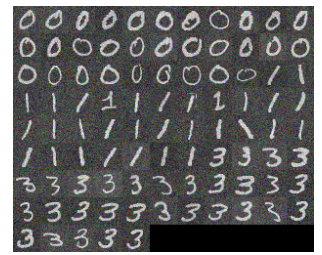

Corrupted DB $(\sigma=40)$

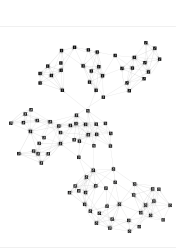

$\mathcal{G}_{10}^{\infty, 0}$

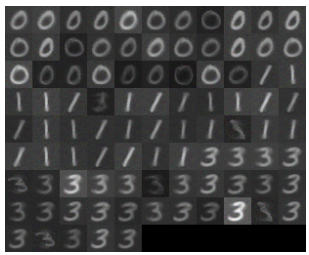

Filtered DB

Fig. 3. $p$-Laplacian regularization of a corrupted image database (with $\tilde{\Delta}_{w}^{p}, p=1$, and $\left.\lambda=10^{-4}\right)$. See text for details.

\begin{tabular}{|c|c|c|c|c|c|c|}
\hline$\sigma$ & \multicolumn{3}{|c|}{20} & \multicolumn{3}{|c|}{40} \\
\hline$\Delta_{w}^{p, *}$ & $\Delta_{w}^{p}$ & $\tilde{\Delta}_{w}^{p}$ & $\Delta_{w}^{p, r w}$ & $\Delta_{w}^{p}$ & $\tilde{\Delta}_{w}^{p}$ & $\Delta_{w}^{p, r w}$ \\
\hline$p=2$ & 14.54 & $\mathbf{1 4 . 6 3}$ & 14.56 & 12.91 & $\mathbf{1 3 . 0 8}$ & 12.88 \\
\hline$p=1$ & 16.37 & $\mathbf{1 6 . 8 3}$ & 16.80 & $\mathbf{1 4 . 0 1}$ & 13.11 & 13.73 \\
\hline
\end{tabular}

Table 1. Image database regularization on directed graphs $\left(\lambda=10^{-4}\right)$. Best results (in terms of PSNR) are bold faced.

\subsection{Meshes}

Finally, we consider 3D colored meshes as a last graph signal and $f: \mathcal{V} \rightarrow \mathbb{R}^{3}$. The meshes are 3D scans from an ancient building and a person. The color is noisy due to the scanning process and the objective is to filter the vertices colors and not their 3D coordinates. The considered graph is a symmetric directed mesh graph (provided from the scan) augmented with a 5-nearest neighbor graph within a 3 -hop. To compare vertices we use $\mathbf{F}_{1}^{\mathbf{f}^{0}}$. However, since the mesh graph is not regular, the feature vectors are not of the same size. We cannot use a $L_{2}$ distance to compare them, so we use instead the Earth Mover Distance between the histograms of $\mathbf{F}_{\mathbf{1}}^{\mathbf{f}^{0}}$. For space contraints, we show results only with $\Delta_{w}^{p, r w}$ that provided the best results. Again with $p=1$ the filtering enables a much better preservation of the signal sharp edges while removing noise. 

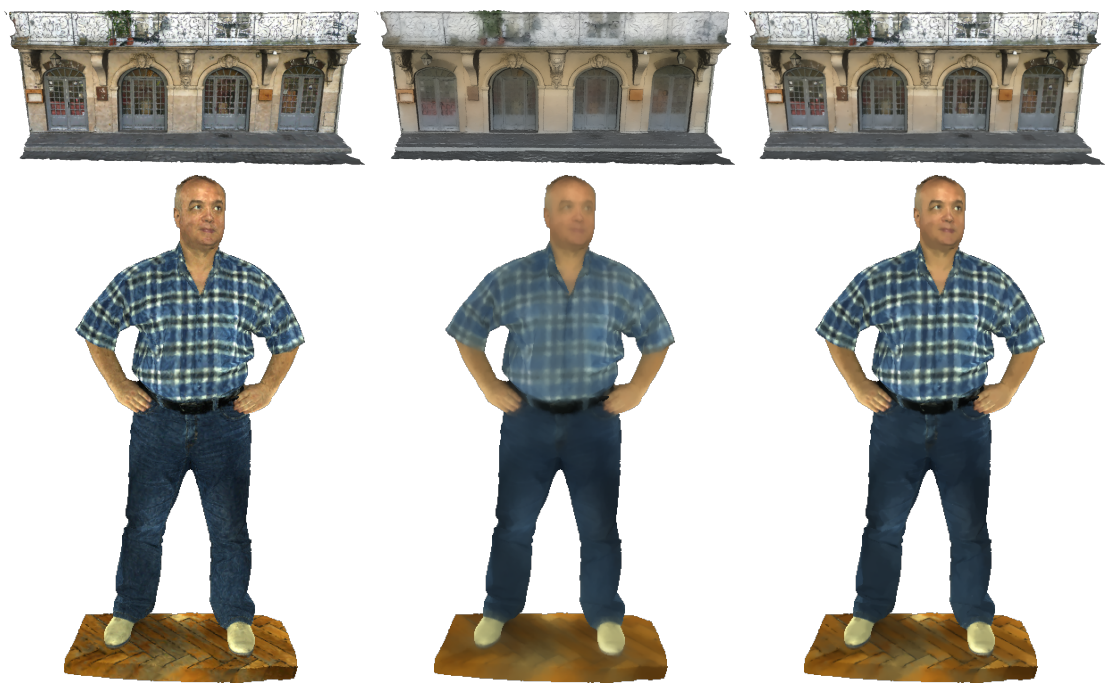

Fig. 4. 3D colored mesh regularization on directed graphs (From left to right: original mesh, filtering with $\Delta_{w}^{p, r w}, \lambda=0.05$, with $p=2$ and $\left.p=1\right)$.

\section{Conclusion}

In this paper we have proposed three formulations for $p$-Laplacians on directed graphs. They used specific difference operators on directed graphs that are inspired from the combinatorial, the normalized and the random-walk laplacians. These formulations of $p$-Laplacians on directed graphs had never been addressed before unless for specific graphs (symmetric and with $p=2[7]$ ). Our proposal goes beyond this. From these formulations, we have considered the problem of $p$-Laplacian regularization of graph signals and proposed a solution to the latter. Finally, some experimental results show the benefit of the approach for the filtering of three types of graph signals: images, images' databases, and 3D colored meshes. Given the results, none of the proposed $p$-Laplacians on directed graphs can be considered as always providing the best results which motivates the need for several formulations. Directed and non symmetric graphs have also shown their interest with respect to classical symmetric undirected graphs. In future works, we will consider other optimization schemes as well as the introduction of non symmetric weights for directed symmetric graphs with applications in image semi-supervised segmentation.

\section{References}

1. Bauer, F.: Normalized graph laplacians for directed graphs. Linear Algebra and its Applications 436(11), 4193-4222 (2012) 
2. Bougleux, S., Elmoataz, A., Melkemi, M.: Local and nonlocal discrete regularization on weighted graphs for image and mesh processing. International Journal of Computer Vision 84(2), 220-236 (2009)

3. Chung, F.R.: Spectral graph theory. CBMS Regional Conference Series in Mathematics 92, 1-212 (1997)

4. Chung, F.: Laplacians and the cheeger inequality for directed graphs. Annals of Combinatorics 9(1), 1-19 (2005)

5. Diestel, R.: Graph Theory, 4th Edition, Graduate texts in mathematics, vol. 173. Springer (2012)

6. Elmoataz, A., Lezoray, O., Bougleux, S.: Nonlocal discrete regularization on weighted graphs: A framework for image and manifold processing. IEEE Trans. Image Processing 17(7), 1047-1060 (2008)

7. Hein, M., Audibert, J., von Luxburg, U.: Graph laplacians and their convergence on random neighborhood graphs. Journal of Machine Learning Research 8, 13251368 (2007)

8. Kheradmand, A., Milanfar, P.: A general framework for regularized, similaritybased image restoration. IEEE Trans. Image Processing 23(12), 5136-5151 (2014)

9. Lézoray, O., Grady, L.: Image Processing and Analysis with Graphs: Theory and Practice. Digital Imaging and Computer Vision, CRC Press / Taylor and Francis (2012)

10. von Luxburg, U.: A tutorial on spectral clustering. Statistics and Computing 17(4), 395-416 (2007)

11. Ng, A.Y., Jordan, M.I., Weiss, Y.: On spectral clustering: Analysis and an algorithm. In: Proceedings of the 14th International Conference on Neural Information Processing Systems: Natural and Synthetic. pp. 849-856 (2001)

12. Olfati-Saber, R., Murray, R.M.: Consensus problems in networks of agents with switching topology and time-delays. IEEE Trans. Automat. Contr. 49(9), 15201533 (2004)

13. Pang, J., Cheung, G.: Graph laplacian regularization for image denoising: Analysis in the continuous domain. IEEE Trans. Image Processing 26(4), 1770-1785 (2017)

14. Shuman, D.I., Narang, S.K., Frossard, P., Ortega, A., Vandergheynst, P.: The emerging field of signal processing on graphs: Extending high-dimensional data analysis to networks and other irregular domains. IEEE Signal Process. Mag. 30(3), 83-98 (2013)

15. Singh, R., Chakraborty, A., Manoj, B.S.: Graph fourier transform based on directed laplacian. In: 2016 International Conference on Signal Processing and Communications (SPCOM). pp. 1-5 (2016)

16. Tremblay, N., Gonçalves, P., Borgnat, P.: Design of graph filters and filterbanks. ArXiv e-prints (2017)

17. Zhou, D., Huang, J., Schölkopf, B.: Learning from labeled and unlabeled data on a directed graph. In: Machine Learning, Proceedings of the Twenty-Second International Conference (ICML 2005), Bonn, Germany, August 7-11, 2005. pp. 1036-1043 (2005)

18. Zhou, D., Schölkopf, B.: Regularization on discrete spaces. In: Pattern Recognition, 27th DAGM Symposium, Vienna, Austria, August 31 - September 2, 2005, Proceedings. pp. 361-368 (2005)

19. Zhou, D., Schölkopf, B., Hofmann, T.: Semi-supervised learning on directed graphs. In: Advances in Neural Information Processing Systems 17 [Neural Information Processing Systems, NIPS 2004, December 13-18, 2004, Vancouver, British Columbia, Canada]. pp. 1633-1640 (2004) 